\title{
Home cage testing of delay discounting in rats
}

\author{
S. KooT \\ Istituto Superiore di Sanità, Rome, Italy \\ and Utrecht University, Utrecht, The Netherlands \\ W. ADRIANI \\ Istituto Superiore di Sanità, Rome, Italy \\ L. SASO \\ Sapienza University, Rome, Italy \\ R. VAN DEN BOS \\ Utrecht University, Utrecht, The Netherlands \\ AND \\ G. LAVIOLA \\ Istituto Superiore di Sanità, Rome, Italy
}

\begin{abstract}
Testing rodents in their home cages has become increasingly popular. Since human intervention, handling, and transport are minimized, behavior can be recorded undisturbed and continuously. Currently existing home cage systems are too complex if only relatively simple operant-learning tests are to be carried out in rats. For that purpose, a new low-cost computer-controlled operant panel was designed, which can be placed inside the home cage. A pilot study was carried out, using an intolerance-to-delay protocol, classically developed for testing behavioral impulsivity. Male adult rats were tested in their home cages, containing the operant panel provided with nosepoking holes. Nose poking in one hole resulted in the immediate delivery of one food pellet (small-soon, SS), whereas nose poking in the other hole delivered five food pellets after a delay (large-late), which was increased progressively each day $(0-150 \mathrm{sec})$. The two daily sessions, spaced $8 \mathrm{~h}$ apart, lasted $1 \mathrm{~h}$ each, and the time-out after food delivery was $90 \mathrm{sec}$. A clear-cut shift toward preference for SS, which is considered a classical index of cognitive impulsivity, was shown at the longest delay. It is noteworthy that rats shifted when the delay interval was longer than the mean intertrial interval — that is, when they experienced more than one delay-equivalent odds against discounting (see Adriani \& Laviola, 2006). The shortened training (2 days) and testing (5 days) phases, as allowed by prolonged and multiple daily sessions, can be advantageous in testing rodents during selected short phases of development. Current research is focusing on further validation of this and similar protocols.
\end{abstract}

Gene-brain-environment interactions are widely studied in animal models, among others, to advance the understanding of how organisms interact with and adapt to their environment and to model human psychiatric disorders. A broad range of test procedures is used for the description and analysis of the behavioral expression of an individual subject - that is, for behavioral phenotyping (De Visser, 2008). However, as far as the study of behavioral phenotyping is concerned, crucial limitations of current approaches are also widely acknowledged (De Visser, van den Bos, Kuurman, Kas, \& Spruijt, 2006; Knapska et al., 2006). Most behavioral tests are restricted to a novel environment and are short-lasting. Furthermore, differences in laboratory environments and human interventions - for example, handling and transport-may compromise the reliability and reproducibility of behavioral data across laboratories (Crabbe, Wahlsten, \& Dudek, 1999; Wahlsten et al., 2003). Recently, much effort has been devoted to developing new and refined methods for behavioral phenotyping. Especially, testing animals in their home cages has become increasingly popular. Since human intervention, handling, and transport are minimized, behavior can be recorded undisturbed and continuously, for prolonged periods of time, as well as in circadian cycles (De Visser et al., 2006; Knapska et al., 2006).

Different types of automated home cage systems have been developed, each having its specific methodology suitable for answering the specific questions of users. For example, the PhenoTyper is a video-based observation system for automation of behavioral tests with singly housed rats or mice, often used, for example, for studying (loco)motor patterns (De Visser, 2008; De Visser et al.,

W. Adriani, walter.adriani@iss.it 
2006). Another system, the IntelliCage, allows automated cognitive and behavioral screening of mice living in social groups - for example, implementing learning tasks (Knapska et al., 2006). Both systems are highly versatile and detailed in monitoring and testing behavior. However, besides being rather costly, these systems are too complex if only relatively simple operant-learning tests are to be carried out (IntelliCage) or if they are currently not able to carry out such operant procedures (PhenoTyper). For that purpose, a new low-cost computer-controlled home cage operant panel (HOP) was developed by joint effort of the Istituto Superiore di Sanità, Rome, and the small company PRS Italia, Rome. This apparatus can be placed inside a standard-size (Macrolon III) cage, enabling the rodent to operate it $24 \mathrm{~h} /$ day.

In order to test the functionality of this panel, a pilot study was carried out, focusing on testing impulsivity. Lack of self-control or impulsive decision making may be an important symptom of psychiatric disorders, such as attention-deficit/hyperactivity disorder (Bechara, 2004; Strubbe \& Woods, 2004). Animal models are crucial in studying the underlying neurobiology of such disorders. In the intolerance-to-delay (ID) task (Evenden, 1999; Evenden \& Ryan, 1999), subjects may choose between a large-late (LL) and a small-soon (SS) reward. Impulsive subjects are intolerant to the forced waiting for the large reward (Evenden \& Ryan, 1996, 1999). Classically, the rats' performance on the ID task is investigated by placing the animals in individual operant chambers for a short period daily (Evenden \& Ryan, 1996, 1999). When the new HOP is used for this task, stress caused by handling and by novelty, which possibly may affect their performance, is prevented. Here, we report the results of a pilot experiment in which this panel was used in an ID task protocol.

\section{METHOD}

\section{Subjects}

Four adult male Wistar rats (Harlan, Italy; mean weight, $429 \mathrm{~g}$ ) were kept in an air-conditioned room (temperature, $21^{\circ} \pm 1^{\circ} \mathrm{C}$; rela- tive humidity, $60 \% \pm 10 \%$ ), on a $12: 12$-h reversed light:dark cycle (lights on at 8:00 p.m.). Prior to the experiments, the animals were housed in pairs in Macrolon III cages. From the start of the protocol, animals were singly housed, with the new apparatus being present in the cage (see below). Water was available ad lib, whereas food (Altromin-R, A. Rieper S.p.A., Vandoies, Italy) was available ad lib until the start of the protocol. The rats had previous experience in impulsivity tasks in a classical Skinner box setting, 2 months prior to the present pilot study.

\section{Apparatus}

The operant-testing apparatus, consisting of four prototype computer-controlled panels (HOP; PRS Italia, Rome; see Figure 1), one for each of the subjects, was placed in a Macrolon III cage with sawdust bedding. The panel contained two nose-poking holes, hole lights, a chamber light, a feeder device, a food magazine where pellets (F0021-J Dustless Precision Pellet 45 mg; Bio-Serv, Frenchtown, NJ) were delivered, and a magazine light. The panel was connected through an interface to a PC, where a specific software (Sca020; PRS Italia, Rome) controlled and recorded all events. Nose poking in one of the two holes of the panel resulted in the delivery of five pellets (large reward, LL), whereas nose poking in the other hole resulted in the delivery of one pellet (small reward, SS). After nose poking and before food delivery, the hole light was turned on for $1 \mathrm{sec}$. Following food delivery, the magazine light was turned on for $90 \mathrm{sec}$, during which time nose poking was recorded but was without scheduled consequences (time-out, TO). The magazine light was then turned off, the chamber light was turned on, and the system was ready for the next trial.

The following variables were recorded automatically: adequate hole poke visits (nose poking after a TO, resulting in the reinforcer delivery) and inadequate hole poke visits (nose poking during the length of the delay or during a TO interval, which were recorded but were without any consequences). For adequate nose poking, the dependent variable was the hole preference (calculated as the percentage of visits at the LL hole over total LL $+\mathrm{SS}$ adequate visits). For inadequate nose poking, the dependent variables were (1) the raw frequency of inadequate visits and (2) the proportion of inadequate to adequate visits. Data were averaged across the two daily sessions and, thus, will be presented as a function of the imposed delay level.

\section{Protocol of the ID Task for Testing Impulsivity}

Two days were required (see Figure 2 ) for pretraining to the experimental protocol. On Day 1, the animals were placed in the cages containing the panel, which occupied one fourth of the total living area. The adaptation period started with $24 \mathrm{~h}$ of access to ad lib regular
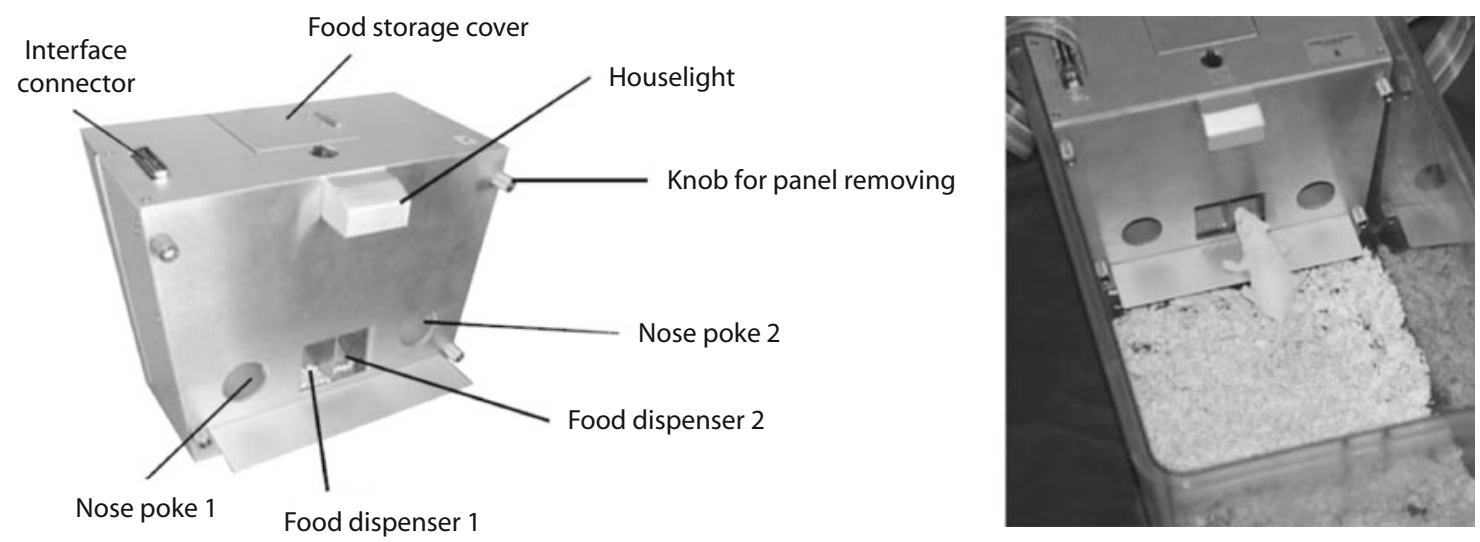

Figure 1. Computer-controlled home cage operant panel (PRS Italia, Rome, Italy) (left), placed in Macrolon III cage (right). 


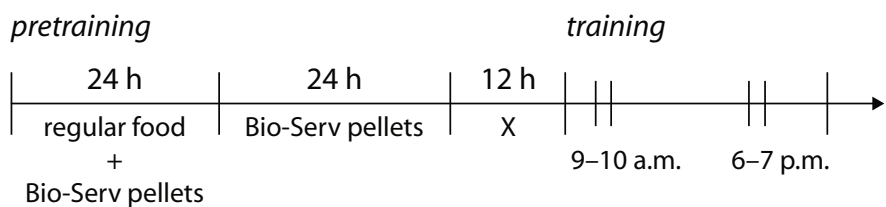

Figure 2. Protocol for home cage testing of impulsivity: pretraining ( 2 days) and first training day. The training lasted just 2 days, whereas the testing phase lasted 5 days. The whole schedule lasted a total of 9 days.

food pellets (Altromin-R) placed in the food rack of the cage top, and, at the same time, rats could obtain Bio-Serv pellets from the panel by nose poking into the holes. On Day 2, the standard (Altromin-R) food was removed for $24 \mathrm{~h}$, while the animals still could obtain precision (Bio-Serv) pellets from the panel. Then, $12 \mathrm{~h}$ of food deprivation followed, in order to increase the rats' motivation to work for food delivery, and the animals entered the training phase of the protocol.

During the subsequent training and testing phases, the animals had access only to precision (Bio-Serv) pellets during the 1-h sessions by operating the panel and had access to a limited amount of standard (Altromin-R) food after each session. Two daily sessions were run, from 9:00 to 10:00 a.m. and 6:00 to 7:00 p.m. (for arguments, see Strubbe \& Woods, 2004). After each session, the total intake of precision pellets was calculated per individual, and additional standard food was given to meet the animals' daily nutritional needs (details available upon request). The end of each session was indicated by switching off all the panel lights, plus the provision of the additional standard pellets in the food rack of the cage top. Training lasted four sessions ( 2 days), until all the subjects reached a significant preference for the large reward.

During the testing phase (10 sessions, 5 days), a signaled delay was added to the 1-sec interval, normally scheduled between nose poking and large-reward delivery. The hole light was kept on during the entire length of this delay. The small-reward delivery was unchanged. Hence, the animals had a choice between an LL and an SS reward. The delay length was fixed for the two daily sessions and was changed over days: $0 \mathrm{sec}$ on the last training day, followed by delays of $15,45,75,105$, and $150 \mathrm{sec}$ on 5 subsequent days. The entire schedule for this protocol lasted 9 consecutive days in total.

In summary, the present ID task was run in tight agreement with previous experiments at our lab (Adriani, Caprioli, Granstrem, Carli, \& Laviola, 2003; Adriani, Della Seta, Dessì-Fulgheri, Farabollini, \& Laviola, 2003; Adriani \& Laviola, 2006; Adriani et al., 2004), with a few minimal changes: First, the TO was elevated to $90 \mathrm{sec}$; second, the system was active daily during two sessions of $1 \mathrm{~h}$ each, rather than during 25-min sessions once daily. These changes allowed an overall reduction in the total duration of the schedule (i.e., only 9 days) and were indeed possible due to the fact that the panel was provided inside the home cage of the subject, rather than in dedicated operant chambers. Of course, this is not the only way an ID task protocol could be run, since many two-choice delayed reinforcement tasks exist. First of all, delay could progress within a session, and the same progression could be repeated across sessions (e.g., Evenden \& Ryan, 1996, 1999). Also, the delay levels could be presented in a randomized, rather than ascending, order or could be titrated until the individual subject expressed indifference in his or her choice (i.e., 50\% SS, 50\% LL; see, e.g., Reynolds, 2006). However, we adopted a gradual increase of delay interval over sessions, since this was done in our previous studies, the results of which were to be compared with the present results.

\section{RESULTS}

Following four training sessions, all the rats showed a significant preference for the large over the small reward (average choice of $94.4 \% \pm 5.3 \%$ for the large reward). Interestingly, instead of showing an exclusive preference (i.e., $100 \%$ for LL), they occasionally chose the alternative SS possibility. This finding, which replicated those of previous experiments in our lab (Adriani, Caprioli, et al., 2003; Adriani, Della Seta, et al., 2003; Adriani \& Laviola, 2006; Adriani et al., 2004), indicates that animals express a constant and active patrolling over the two alternative possibilities, as they adaptively probe whether there is any change over time in the outcome associated with nose poking at the nonpreferred hole.

When delays were gradually increased over days (Figure 3), rats showed a shift toward SS choices at the longest delays (cf. Evenden \& Ryan, 1996, 1999). A flatter or steeper shift toward SS choice is a classical index of reduced or increased impulsivity, respectively (Adriani \& Laviola, 2006; Evenden \& Ryan, 1996, 1999). Indeed, subjects may be individually characterized by delayavoidant traits or, vice versa, show an ability to cope with the delay constraint (Evenden, 1999). Accordingly, in all mammals, the most impatient among subjects are termed impulsive, in that they display intolerance to the forced waiting for a delayed reward. Thus, average group profiles are not entirely informative (Adriani, Caprioli, et al., 2003; Adriani et al., 2004), and precious information may emerge when individual profiles are considered. Indeed,

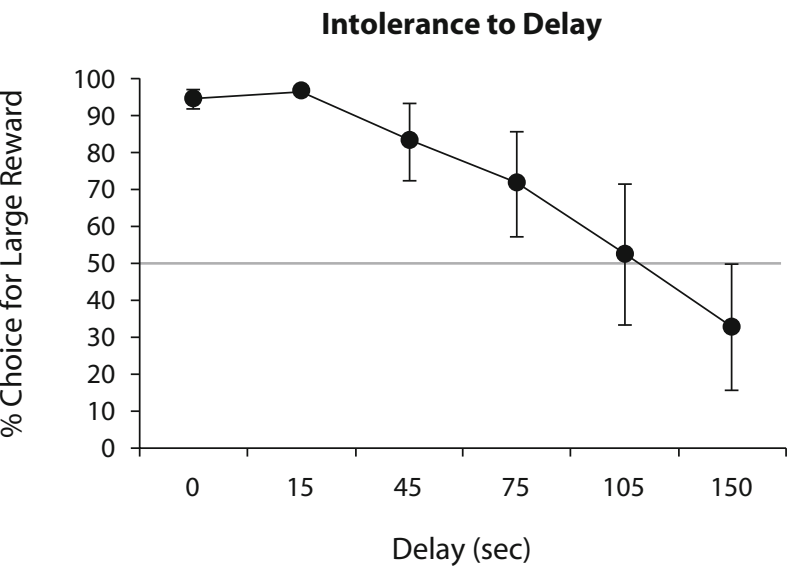

Figure 3. Choice behavior by rats $(n=4)$ tested with the intolerance-to-delay protocol, shown during daily sessions in the home cage situation. Data represent the mean $( \pm S E M)$ choice per day (as a percentage) for the larger reward, delivered after a delay. 
each individual rat showed its own steepness profile (Figures 3 and 4), with Rat 1 showing nearly no shift and Rat 2 being the most impulsive one.

Because nose poking in either hole during the course of the delay interval had no scheduled consequences, it was termed an inadequate response (Sagvolden, 2000; Sagvolden \& Sergeant, 1998). This measure provides an index of the (in)ability to inhibit an unnecessary response. Data for delay $=0$ reveal that inadequate responding was $67.6 \%$ of total nose poking, as if a couple of ineffective nose pokes were expressed per TO. More precisely, if we admit that ineffective nose pokes were released in bouts of two, then rats emitted a mean of 20.2 pairs of ineffective nose pokes per session, spaced an average of $106.2 \mathrm{sec}$ apart. Such a long interresponse interval provides a precious internal control for rats' understanding of TOs as windows of unavailability for their operant device. As the length of the delay was increased, inadequate nose pokes at the LL hole peaked and then decreased, whereas nose pokes at the SS hole progressively increased (Figure $5 \mathrm{~A}$ ). Such a finding suggests that, during the length of the delay interval, when they had to wait for the large reward to be delivered, the rats were (ineffectively) demanding more and more the smaller, immediate reward. However, the rate of ineffective nose pokes during delay intervals did not rise as delays were increased: If we compare delay $=15 \mathrm{sec}$ with delay $=150 \mathrm{sec}$, when the waiting-constraint of delay was tenfold, it is clear that the SS ineffective nose poking was just doubled, and the total SS + LL inadequate visits were even slightly reduced. Interestingly, the percentage of inadequate nose pokes out of the total nose pokes remained fairly stable for each hole (around an overall mean of 75.3\%; see Figure 5B). Since the number of effective choices for SS was increasing also, as Figures 3 and 4 indicate, both effective and ineffective SS nose poking apparently rose at a similar pace. As a consequence, the rats showed no specific in-

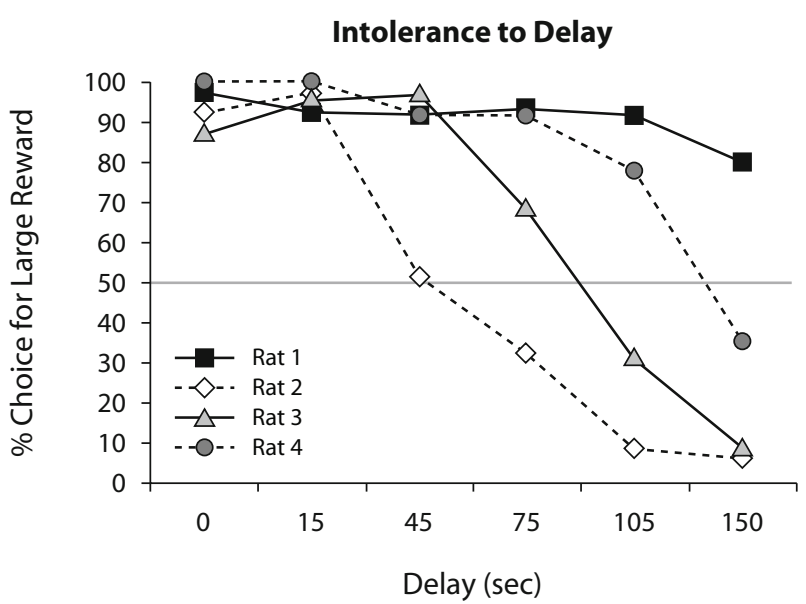

Figure 4. Choice behavior by 4 individual rats tested with the intolerance-to-delay protocol, shown during daily sessions in the home cage situation. Data represent the choice per day (as a percentage) for the larger reward, delivered after a delay. crease in the rate of inadequate nose poking at the SS hole during the whole testing period, thus indicating no sign of motor impulsivity that could well have been released by this task.

\section{DISCUSSION}

The aim of the present pilot was to test a new prototype of computer-controlled HOPs, currently designed to be used for testing impulsivity of rats (and mice) in their home cages. This pilot experiment showed that, in principle, it is possible to measure impulsive choice behavior in rats within a home cage setting. As was expected, when delays increased over days, rats showed a shift toward preference for SS at the longest delays. Similar results have been found in previous studies (cf. Adriani, Caprioli, et al., 2003; Adriani, Della Seta, et al., 2003; Adriani \& Laviola, 2006). To be able to compare the present results with those in previous studies, it is necessary to recalculate the outcomes with respect to TO duration. Classical definitions of impulsivity predict that a given factor (in the present study, delay) will discount the perceived value of the large reward, whereas the attractiveness of the small reward is unchanged. In the case of a very strong discounting process, which is warranted by the negative emotional impact of that factor (i.e., by the delay-generated aversion), animals will eventually express a preference for the SS reward. Such an impulsive choice is suboptimal and antieconomical, by definition, in that it leads to a lesser amount of food being gained in the long term (Evenden, 1999; Ho, Mobini, Chiang, Bradshaw, \& Szabadi, 1999; Monterosso \& Ainslie, 1999). Indeed, if preference shift from LL toward SS is convenient, it will be supported by "rational" criteria of payoff maximization. Thus, only decisions that are taken against economic convenience can be considered as an unbiased and reliable impulsivity index in the field of decision making (Adriani \& Laviola, 2006, 2009).

It may be useful to introduce the so-called indifferent point, at which either choice is mathematically identical in terms of total foraging, since it helps to clarify the economical aspects of the task. By definition, the long-term outcome is indifferent when rats can choose to receive either five SS deliveries of one pellet each or a single LL reward of five pellets during comparable intervals of time. As outlined by Adriani and Laviola (2006), the crucial unit to account for temporal features within the ID task is represented by the mean intertrial interval (mITI), composed of the TO interval (scheduled after food delivery) plus the spontaneous waiting by the animal (expressed after the end of TO and before the next nose poking). After each TO of $15 \mathrm{sec}$, animals performed the next nose poking after $10 \mathrm{sec}$, on average (Adriani \& Laviola, 2006); thus, a fundamental descriptor of temporal features within the task, which could also be labeled crucial time unit, is represented by the task-specific parameter of mITI $=25 \mathrm{sec}$. Animals will, in fact, experience the same overall payoff either if they choose five consecutive SSs, during a time window lasting a total of five mITIs $(5$ times $25 \mathrm{sec}=$ $125 \mathrm{sec}$ ) or if they choose LL just once and afford a sole 
A

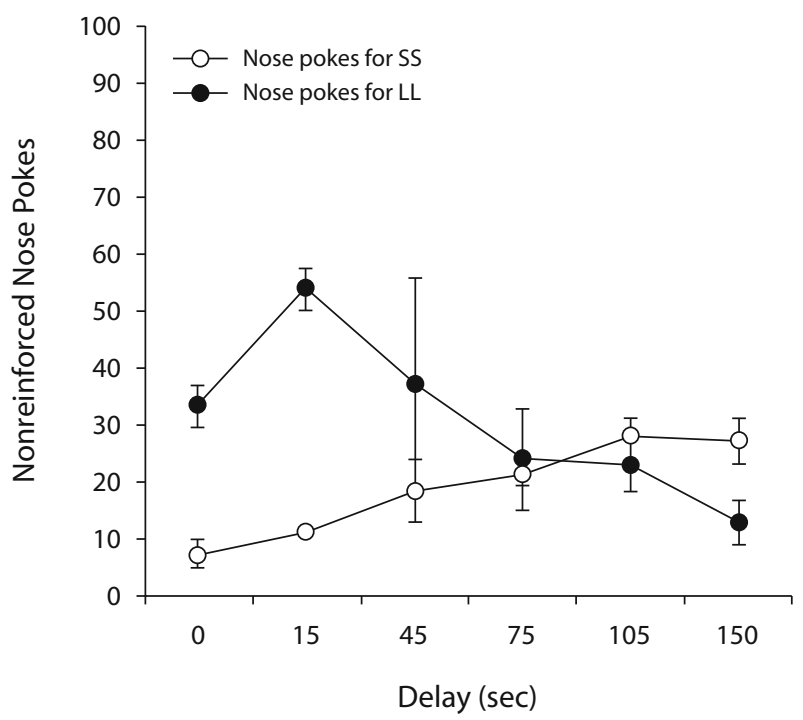

B

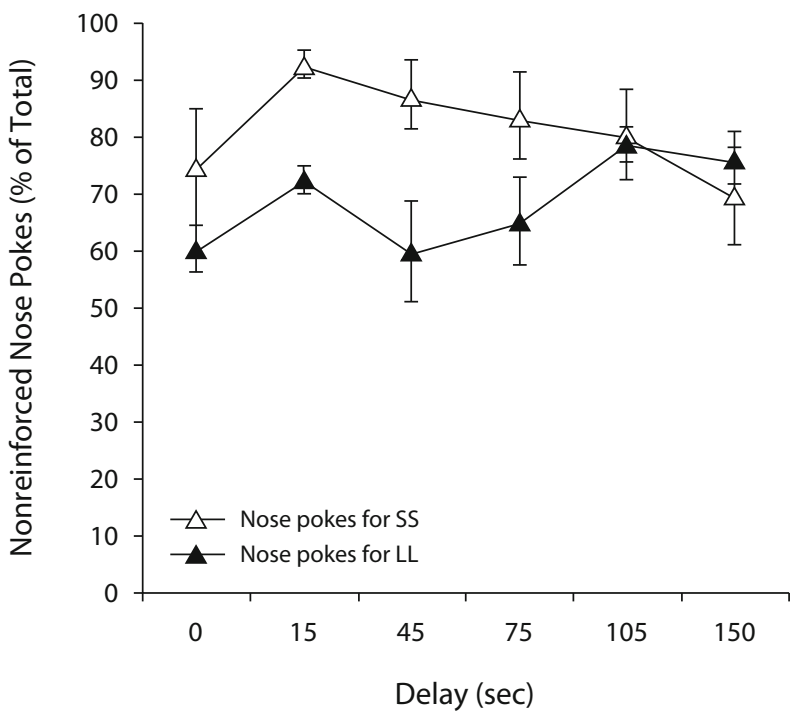

Figure 5. Inadequate responding at both the small-soon (SS) and large-late (LL) holes (i.e., nose poking during the length of the delay interval being without any consequence) shown by rats $(n=4)$ tested in the intolerance-to-delay protocol. (A) Mean $( \pm S E M)$ absolute quantity of inadequate nose pokes per session. (B) Mean $( \pm S E M)$ percentages of inadequate nose pokes out of the total number of nose pokes.

delay, whose length is four times the mITI (i.e., a 100-sec delay) plus a further $25 \mathrm{sec}$ (i.e., its own TO followed by spontaneous waiting).

In the previous study, where the ID task was run inside the classical Skinner box setting, the TO interval was set at $15 \mathrm{sec}$, the mITI was $25 \mathrm{sec}$, and thus the indifferent point was encountered at a delay of $100 \mathrm{sec}$. In the present work, we set a TO of $90 \mathrm{sec}$, so that mITI was $100 \mathrm{sec}$, and the indifferent point was encountered, on average, at a delay of $400 \mathrm{sec}$. The reason for a shorter TO in previous studies resided mainly in shorter sessions, which are inherent to classical Skinner box testing. Indeed, when experiments with tens of subjects are run over several days, the experimenter needs to test several subjects per day in the same box. Thus, sessions usually range between 20 and $30 \mathrm{~min}$, and the TO ranges between 15 and $25 \mathrm{sec}$. This allows animals to be presented with around 60 opportunities to make SS/LL selections. Animals living permanently within the reach of the operant panels will be offered the same quantity of selection opportunities while longer sessions are run. This can easily be done by using longer TO intervals, which obviously imply a longer mITI. Hence, the reinforcing rate inherent to the task was slower in the present home cage setting than it is in classical conditions, and this perhaps explains why longer delays were apparently tolerated by the rats. As a matter of fact, the present setting provided the rats with more time to elaborate their strategy, which is not the case with classical sessions. This is another advantage of home cage testing.

In brief, for the present pilot work, receiving a single LL reward of five pellets after a delay of $400 \mathrm{sec}$ would actually have been indifferent with respect to receiving five SS deliveries of one pellet each, with four mITIs averaging $100 \mathrm{sec}$ between them. Note that, in the previous study, receiving a single LL reward of five pellets after a delay of $100 \mathrm{sec}$ was indifferent with respect to receiving five deliveries of one pellet each, with four mITIs averaging $25 \mathrm{sec}$ between them. This notion helps us to compare the present results with those in the previous study (Adriani \& Laviola, 2006), where we proposed that the impact of a given delay could be expressed as a (sub)multiple of the mITI. By referring to delay-equivalent odds against discounting, using the formula odds $=$ delay/mITI, the fact that mITI was fourfold in the home cage versus the classical setting implies that the present delays of the ID task should be divided by four. As such, the subjective indifference between LL and SS and the development of an SS preference, respectively, were clearly observed in the present pilot study at delays of 105 and $150 \mathrm{sec}$ (odds $=1.05$ and 1.50), which would therefore correspond to $26.25 \mathrm{sec}(=105 / 4)$ and $37.50 \mathrm{sec}(=150 / 4)$ of the previous study (Adriani \& Laviola, 2006). In that study, a shift in preference was found between delays of 30 and $45 \mathrm{sec}$ (equivalent odds $=1.2-1.8$ ). Thus, a reference to the delay-equivalent odds values renders delay lengths fairly comparable across studies, even though a further validation should be carried out. Currently, there is rising interest in comparing directly the results obtained from home cage testing versus the conventional Skinner box setting. Furthermore, the two kinds of setting will not necessarily lead to identical results: As underlined by a very recent report (Caprioli et al., 2009), comparing the performance of animals in their home cage versus a different testing apparatus may open avenues for very innovative findings. 
Apart from the rewarded nose pokes, unrewarded nose poking (i.e., during the length of the delay interval, when it had no scheduled consequences; Morgan, 1972) was recorded. This inadequate responding has been proposed as an index of inability to inhibit an unnecessary response - a measure of restlessness, in other words (Sagvolden, 2000; Sagvolden \& Sergeant, 1998). The present results confirm a progressive elevation in absolute quantities (i.e., raw frequency of nose pokes) for SS inadequate responding (Adriani, Della Seta, et al., 2003). These data should be looked at carefully, since they might suggest a rise of restlessness, generated by the progressive increase of delay intervals. However, when we consider the relative proportion of inadequate nose pokes out of the total number of nose pokes, this parameter apparently shows a rather stable profile over the entire testing period, or even a slight nonsignificant decrease as delays got longer (Figure 5B). Thus, we conclude that, by exploiting the signaling cues provided by the panel, the rats were able to distinguish between specific portions of time - that is, when the panel was effectively available, as opposed to the delay-long windows of ineffectiveness. Indeed, if their response was random or aspecific and/or motor restlessness was generated by the waiting constraint, an increasing proportion of inadequate visits would emerge along the whole session. The present data, actually supporting a constant or even decreasing proportion of inadequate visits, indicate that rats refrained somewhat from nose poking after having expressed their choice.

Furthermore, since we propose that no specific increase in restlessness was generated by the progression of delay intervals, the corollary is that cognitive, rather than motoric, impulsivity was assessed in the present study. The shift to SS was not a side effect of enhanced and aspecific SS nose poking during panel ineffectiveness. To study restlessness in more detail, other protocols should be implemented. For instance, in studies with spontaneously hyperactive and impulsive rats (Johansen, Sagvolden, \& Kvande, 2005), ineffective responding was punished somewhat, in that it led to resetting of delay length. In this kind of protocol, the real duration of delay can be dependent on rats' own behavior. It is hence difficult to determine the effect of the delay interval alone (Johansen et al., 2005), since longer delays consist mainly of effective responses followed by short interresponse times. It is beyond the limits and scope of this article to discuss this issue in more detail. In our hands, the present ID task allows the possibility of extracting data on rewarded versus unrewarded nose pokes, which can respectively be used to distinguish between cognitive and motor impulsivity (Adriani, Caprioli, et al., 2003; Adriani, Della Seta, et al., 2003; Evenden, 1999).

\section{Advantages and Disadvantages of Home Cage Testing}

As compared with the classical Skinner box setting, a couple of advantages of home cage testing can be distinguished. First of all, two factors of the conventional proto- col are clearly diminished - that is, the stress of handling and the novelty experience caused by transport to the test apparatus for several days. However, it is as yet unclear to what extent this effect can be traced back in the results. Second, prolonged training sessions are allowed in the home cage, thus shortening considerably the duration of the training period - that is, 4 days in the present protocol versus 1 week in the standard protocol (Adriani, Caprioli, et al., 2003; Adriani, Della Seta, et al., 2003; Adriani \& Laviola, 2006). A short training period is especially desirable for testing developing rodents, since developmental stages may last only 1 or 2 weeks for rats and mice (e.g., see Adriani, Caprioli, et al., 2003; Adriani \& Laviola, 2003). As for the testing period, in the present pilot study, the same delay was tested twice a day, since it was unclear whether early and late sessions during the dark hours would impose a bias due to circadian rhythm fluctuations. Further studies could address more specifically and might lead to a shortened schedule as well. Another important advantage of the use of the HOP, as compared with other home cage systems, is its simplicity in use and relative low cost. HOPs can be placed in each standard Macrolon III cage, allowing one to house animals in common animal rooms, without any special requirements except for cables to an interface that then connects the panels to the controlling computer.

Difficulties and disadvantages of the HOP can be found as well. In the present protocol, rats must be housed individually throughout the entire protocol duration. Stress through social isolation influences the reward system (Hall, Humby, Wilkinson, \& Robbins, 1997; Van den Berg et al., 1999) and decision making as such, as well. Impulsivity tasks are particularly interesting during adolescence, but social deprivation may produce changes in reward sensitivity (Van den Berg et al., 1999), as well as psychotic symptoms (Leussis \& Andersen, 2008), particularly during this ontogenetic period. Solutions for this might be found in (partially) social housing, combined with technologies such as transponder-operated access to the operant panel(s) and/or communal cages. However, these adaptations to the model would involve extra animal training and might compromise the simplicity and easy use of the present system. The HOPs are placed in standard Macrolon III cages inside a standard animal room. Unlike the classical Skinner boxes, which are surrounded by soundproof boxes, no visual, audio, or olfactory barriers are used in the present system. It is as yet unclear to what extent individuals can influence each other's choice behavior-for example, by making food calls.

\section{Conclusion}

The present pilot study showed the possibility of testing impulsivity in the home cage, using an operant panel. In theory, other protocols can be run with this panel as well. For example, we recently proposed that a yoked probabilistic-discount (PD) protocol may exist for each ID task, as validated using the classical Skinner box setting (Adriani \& Laviola, 2006). Thus, beside the present home cage ID task, a corresponding PD task has been 
run for investigating gambling behavior. Adult male rats were housed, trained, and tested as described in the present pilot study. However, during the training phase, large reward delivery was randomly released or omitted, by setting a percentage of probability $(\mathrm{P}=$ releases/ demands $* 100$ ). The level of $P$ was fixed for daily sessions and decreased progressively every other day, thus implementing a large-reward rarefaction (Adriani \& Laviola, 2006). When the P level was decreased, some rats reduced their LL preference only slightly, and some other rats developed a clear preference for SS. According to Madden, Ewan, and Lagorio (2007), indeed, there is a clear relationship between delay and probability discounting, in that the same individual aversion to delayed rewards comes along with greater value being attributed to unpredictable than to predictable rewards. Similarly, impulsive and/or gambling behavior is more likely to result from a reduced self-control ability, rather than from simplistic value-discounting functions (Monterosso \& Ainslie, 1999).

Current research is focusing on the further validation of both ID and PD task home cage protocols, as well as on finding solutions for the above-mentioned limitations. For instance, it could be informative to determine impulsivity curves generated when the home cage ID task is run under several possible combinations of TO duration and session length. We assume, indeed, that the same individual would show more delay (in)tolerance if facing long (or short) sessions, whereas the role of TO has yet to be explored. Rats' spontaneous waiting after a TO and before their next response (response time, RT) could be differentially affected if they are faced with a short versus long TO after food delivery. If, indeed, rats track and do adapt to a task-specific reinforcing rate (Gallistel \& Gibbon, 2000; Podlesnik \& Shahan, 2008), their mITI average value should be critically paced by both the imposed TO duration and adaptive development of an individual RT value. Therefore, it would be valuable to further refine the present home cage ID task, as well as to validate other types of ID task protocols.

\section{AUTHOR NOTE}

Research was performed along the lines of the "ADHD-sythe" young investigator project (to W.A.): "under-40 call" by the Italian Ministry of Health (assigned), and "ERC-StG" by EU-FP7-Ideas (pending). It was also supported by the bilateral Italy-U.S. Program on Rare Diseases (7NR1) and by the ERARE-EuroRETT Network (ERAR/6), EU (to G.L.). S.K. was a recipient of an Erasmus fellowship, within the Lifelong Learning Programme (to L.S.), supporting her stay at the Istituto Superiore di Sanità. The authors thank Pietro Serenellini (PRS Italia) for technical design and support. Correspondence concerning this article should be addressed to W. Adriani, Behavioral Neuroscience Section, Department of Cell Biology and Neurosciences, Istituto Superiore di Sanità, Viale Regina Elena 299, Rome 1-00161, Italy (e-mail: walter .adriani@iss.it).

Note-This article is based on a presentation made at Measuring Behavior 2008.

\section{REFERENCES}

Adriani, W., Caprioli, A., Granstrem, O., Carli, M., \& Laviola, G. (2003). The spontaneously hypertensive-rat as an animal model of
ADHD: Evidence for impulsive and nonimpulsive subpopulations. Neuroscience \& Biobehavioral Reviews, 27, 639-651.

Adriani, W., Della Seta, D., Dessì-Fulgheri, F., Farabollini, F., \& LAVIOLA, G. (2003). Altered profiles of spontaneous novelty seeking, impulsive behavior, and response to $d$-amphetamine in rats perinatally exposed to bisphenol A. Environmental Health Perspectives, 111, 395-401.

Adriani, W., \& Laviola, G. (2003). Elevated levels of impulsivity and reduced place conditioning with $d$-amphetamine: Two behavioral features of adolescence in mice. Behavioral Neuroscience, 117, 695-703.

AdRiani, W., \& LaVIola, G. (2006). Delay aversion but preference for large and rare rewards in two choice tasks: Implications for the measurement of self-control parameters. BMC Neuroscience, 23, 52.

Adriani, W., \& Laviola, G. (2009). Animal models and mechanisms of impulsivity in adolescence. In T. Palomo, R. Beninger, T. Archer, \& R. Kostrezwa (Eds.), Beyond neuropsychiatric diagnostics: Symptoms not disorders (Strategies for Studying Brain Disorders, Vol. 9, p. 385434) Madrid: Editorial CYM.

Adriani, W., Rea, M., Baviera, M., Invernizzi, W., Carli, M., GhiRARDI, ET AL. (2004). Acetyl-L-carnitine reduces impulsive behavior in adolescent rats. Psychopharmacology, 176, 296-304.

BECHARA, A. (2004). The role of emotion in decision-making: Evidence from neurological patients with orbito-frontal damage. Brain \& $\mathrm{Cog}$ nition, 55, 30-40.

Caprioli, D., Celentano, M., Dubla, A., Lucantonio, F., NenCINI, P., \& BAdiani, A. (2009). Ambience and drug choice: Cocaineand heroin-taking as a function of environmental context in humans and rats. Biological Psychiatry, 65, 893-899.

Crabbe, J. C., Wahlsten, D., \& Dudek, B. C. (1999). Genetics of mouse behavior: Interactions with laboratory environment. Science, 284, 1670-1672.

De VISSER, L. (2008). Home sweet home: Home cage testing for behavioral phenotyping of mice. Unpublished doctoral thesis, Utrecht University, The Netherlands.

De Visser, L., van den Bos, R., Kuurman, P. W. W., Kas, M. J. H., \& SPRUiJT, B. M. (2006). Novel approach to the behavioral characterization of inbred mice: Automated home cage observations. Genes, Brain \& Behaviour, 5, 458-466

Evenden, J. L. (1999). Varieties of impulsivity. Psychopharmacology, 146, 348-361.

Evenden, J. L., \& Ryan, C. N. (1996). The pharmacology of impulsive behavior in rats: The effects of drugs on response choice with varying delays of reinforcement. Psychopharmacology, 128, 161-170.

EVENDEN, J. L., \& RYAN, C. N. (1999). The pharmacology of impulsive behavior in rats: VI. The effects of ethanol and selective serotonergic drugs on response choice with varying delays of reinforcement. Psychopharmacology, 146, 413-421.

Gallistel, C. R., \& Gibbon, J. (2000). Time, rate, and conditioning. Psychological Review, 107, 289-344.

Hall, F. S., Humby, T., Wilkinson, L. S., \& Robbins, T. W. (1997). The effects of isolation-rearing on sucrose consumption in rats. Physiology \& Behavior, 62, 291-297.

Ho, M. Y., Mobini, S., Chiang, T. J., Bradshaw, C. M., \& Szabadi, E. (1999). Theory and method in the quantitative analysis of "impulsive choice" behaviour: Implications for psychopharmacology. Psychopharmacology, 146, 362-372.

Johansen, E. B., Sagvolden, T., \& Kvande, G. (2005). Effects of delayed reinforcers on the behavior of an animal model of attentiondeficit/hyperactivity disorder (ADHD). Behavioural Brain Research, 162, 47-61.

Knapska, E., Walasek, G., Nikolaev, E., Neuhäusser-Wespy, F., LipP, H. P., KaczmareK, L., \& Werka, T. (2006). Differential involvement of the central amygdala in appetitive versus aversive learning. Learning \& Memory, 13, 192-200.

Leussis, M. P., \& ANDERSEN, S. L. (2008). Is adolescence a sensitive period for depression? Behavioral and neuroanatomical findings from a social stress model. Synapse, 62, 22-30.

Madden, G. J., Ewan, E. E., \& Lagorio, C. H. (2007). Toward an animal model of gambling: Delay discounting and the allure of unpredictable outcomes. Journal of Gambling Studies, 23, 63-83.

Monterosso, J., \& Ainslie, G. (1999). Beyond discounting: Possible 
experimental models of impulse control. Psychopharmacology, 146, 339-347.

MoRgan, M. J. (1972). Fixed-ratio performance under conditions of a delayed reinforcement. Journal of Experimental Psychology: Animal Behavior Processes, 17, 95-98.

Podlesnik, C. A., \& Shahan, T. A. (2008). Response-reinforcer relations and the resistance to change. Behavioral Processes, 77, 109-125.

REYNOLDS, B. (2006). A review of delay-discounting research with humans: Relations to drug use and gambling. Behavioral Pharmacology, 17, 651-667.

SAGVOLDEN, T. (2000). Behavioral validation of the spontaneously hypertensive rat (SHR) as an animal model of attention deficit/ hyperactivity disorder (AD/HD). Neuroscience \& Biobehavioral Reviews, 24, 31-39.

Sagvolden, T., \& Sergeant, J. A. (1998). Attention deficit/ hyperactivity disorder: From brain dysfunctions to behavior. Behavioural Brain Research, 94, 1-10.

Strubbe, J. H., \& Woods, S. C. (2004). The timing of meals. Psychological Review, 111, 128-141.

Van den Berg, C. L., Pijlman, F. T., Koning, H. A., Diergaarde, L., Van Ree, J. M., \& Spruitt, B. M. (1999). Isolation changes the incentive value of sucrose and social behavior in juvenile and adult rats. Behavioural Brain Research, 106, 133-142.

Wahlsten, D. M., Phillips, T. J., Boehm, S. L., II, Burkhart/ Kasch, S., Dorow, J., Doerksen, S., ET AL. (2003). Different data from different labs: Lessons from studies of gene-environment interaction. Journal of Neurobiology, 54, 283-311.

(Manuscript received October 31, 2008; revision accepted for publication May 7, 2009.) 